\title{
Nanoscale Probing of Electrode Surfaces by Scanning Force Microscopy
}

\author{
David J. Fermín*
}

\begin{abstract}
The development of increasingly sophisticated hierarchical structures in the design of functional electrode surfaces introduces a high degree of complexity into the analysis of conventional electrochemical responses. This article briefly summarizes recent developments in techniques based on the concepts of scanning force microscopy (SFM) for the in situ characterization of electrode surfaces. High-resolution morphological studies during phase formation and ion insertion processes have provided valuable insights into the correlations between electrochemical signals and surface structural changes. Novel measuring modes such as the combined scanning electrochemical microscopy-SFM have opened the possibility of mapping electrochemical reactivity of heterogeneous surfaces at the sub-micron range. Finally, the use of SFM for accurate analysis of electrostatic forces associated with the electrochemical double layer is also highlighted.
\end{abstract}

Keywords: Double layer forces · Electrochemistry · Modified electrodes · Scanning electrochemical microscopy

\section{Introduction}

Current trends in the design of sensing devices, systems for energy conversion, and molecular electronics heavily rely on the assembly of functional materials at electrode surfaces. Supramolecular structures, nanostructured materials, redox/conducting polymers and biomolecular components are being increasingly used in sophisticated hierarchical architectures. Electrochemical techniques have the unique advantages of finely controlling the redox state of the systems and monitor the flux of charges associated with electron transport through

\footnotetext{
${ }^{*}$ Correspondence: Prof. Dr. D.J. Fermín

Departement für Chemie und Biochemie

Universität Bern

Freiestrasse 3

$\mathrm{CH}-3012$ Bern

Tel: +41316314317

Fax: +41316313994

E-Mail: david.fermin@iac.unibe.ch
}

the whole assembly. However, complex structures may exhibit a degree of disorder which makes the interpretation of current-potential relationships rather complicated. Therefore, techniques able to resolve morphological and chemical properties of surfaces at the molecular scale are highly valuable tools for the characterization of modified electrodes.

Techniques based on scanning force microscopy (SFM) have had a major impact in the study of electrochemical interfaces, particularly in systems featuring polymer films, mesoscopic phases, and self-assembled nanostructures [1]. As the tip-substrate interactions are not governed by electron tunnelling processes as in STM, conventional in situ topographic analysis of electrode surfaces can be performed with commercially available tips used for measurements in liquid environment. Furthermore, the availability nowadays of various measuring modes such as friction force microscopy, phase imaging, and energy dissipation chemical force allow high-resolution mapping of visco-elastic and rheological properties of materials at electrode surfaces [2-5]. In this article, the impact of SFM-based techniques as tools for the characterization of electrode surfaces is briefly reviewed. The discussion will not only be focused on the power of SFM for mapping the morphology of electrode surfaces, but also on the capabilities for imaging the electrostatic forces associated with the double layer and the local electrochemical reactivity at the sub-micron scale.

\section{Topographic Studies of Electrode Surfaces}

Intense debates in the early days of SFM were centred on the issue of whether truly atomic resolution can be obtained from this approach. In the case of STM, the characteristic distance dependence of electron tunnelling inherently increases the probability that this phenomenon is determined by true atom-atom interactions between the tip and the substrate. Considering the sharper distance dependence of the atom-atom forces (e.g. van der Waals forces), it would have been expected that SFM resolution will be even higher than in STM. However, two aspects introduce strong limitations to the image resolution: (i) measurable forces concentrated in few atoms generate strong deformation of the tip and/or substrate and (ii) long-range forces (e.g. electrostatic forces) are sensitive to the overall shape of the tip. The second argument appears to be especially relevant in electrochemical systems in which variations of the applied potential may introduce substantial changes in the charge of the diffuse layer (this point will be further discussed in Section 3.3).

Despite these limitations in the SFM resolution, Gewirth and co-workers reported periodic features related to atomically resolved images of $\mathrm{Au}(111)$ surfaces during the electrodeposition of $\mathrm{Cu}$ [6]. As illustrated in Fig. 1, high-resolution images revealed lattice spacing of $\mathrm{Cu}$ adatoms of $0.29 \pm 0.02 \mathrm{~nm}$ in electrolytes containing perchloric acid, while $0.49 \pm 0.02 \mathrm{~nm}$ was 

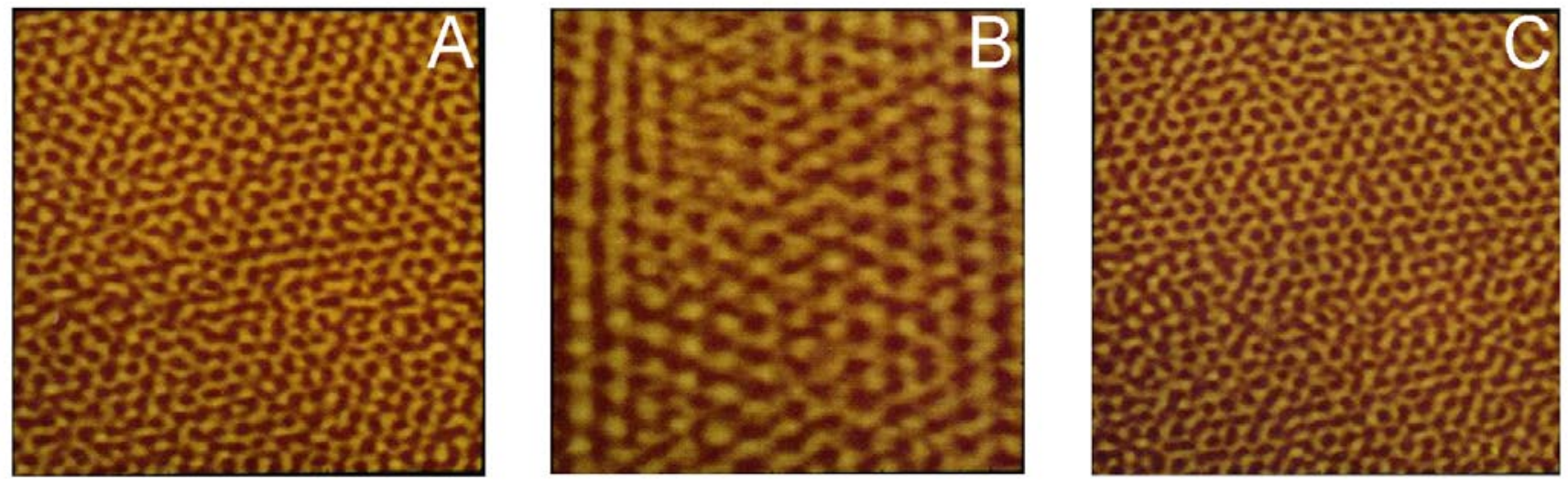

Fig. 1. In situ contact mode SFM images $(6 \mathrm{~nm} \times 6 \mathrm{~nm})$ of a $\mathrm{Cu}$ overlayer on a $\mathrm{Au}(111)$ surface at $(\mathrm{A}) 0.11 \mathrm{~V}$ in perchloric acid solution and (B) $0.14 \mathrm{~V}$

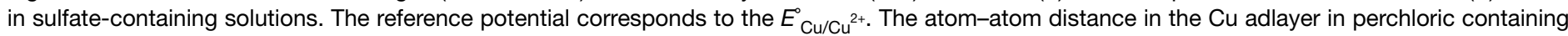
solution (A) was $0.26 \mathrm{~nm}$, suggesting the presence of an incommensurate structure of closely packed Cu atoms. On the other hand, the image in sulphate electrolyte (B) showed a more open structure featuring $0.49 \mathrm{~nm}$ atom spacing. This arrangement is consisting with a ( $\sqrt{ } 3 \times \sqrt{ } 3$ ) $\mathrm{R} 30^{\circ}$ structure presumably influenced by the co-adsorption of sulphate ions. The image $(\mathrm{C})$ shows the bulk-deposited $\mathrm{Cu}$ at $-0.1 \mathrm{~V}$. A lattice spacing of $0.26 \mathrm{~nm}$ was observed in the presence of both electrolytes. Reprinted with permission from ref. [6]. Copyright (1991) AAAS.

observed in sulphate electrolyte. Towards the potential for $3 \mathrm{D}$ nucleation, the lattice spacing is reduced to $0.26 \pm 0.02 \mathrm{~nm}$ in both electrolytes. Alves and Porter also reported high-resolution images of fluorinated alkanethiolate monolayer at Au (111) surfaces featuring a $(2 \times 2)$ adlayer with distances of $0.58 \pm 0.02 \mathrm{~nm}$ and $1.01 \pm 0.02 \mathrm{~nm}$ for the nearest-neighbour and the next-nearestneighbour [7]. This molecular arrangement correlates rather well with the electrochemical responses originating from the cathodic desorption of the thiol monolayer.

These two classical examples illustrated that SFM technique can yield atomic and molecular resolution of electrode surfaces. However, it was still unclear how this resolution can be reconciled with the nanometric size of the tips. Oden, Tao and Lindsay elegantly addressed this issue by studying the reconstructed $\mathrm{Au}$ (111) surface under electrochemical control by STM and SFM [8]. Although STM consistently provided higher resolution imaging than SFM, the latter also showed periodic atomic-like features at high resolution. The observed features as well as the potential range for lifting the reconstruction were relatively consistent for both techniques, confirming that the periodic structures have the same origin. Curiously, SFM tips exhibiting poor resolution in the $10 \AA$ range did show atomic resolution with the same applied force. This report clearly demonstrated that the atomic scale features in SFM originate from the periodic interactions between tip and substrate rather than a truly atom-atom interaction.

The most common application of SFM in electrochemistry consists of establishing correlation between structure and reactivity of modified electrodes. For instance, Naoi et al. also reported clear correlation between the topography of electrogenerated polypyrrole films in the presence of micelles and its redox properties [9]. Micellar solutions of sodium dodecyl sulphate and dodecylbezene sulfonate effectively template the formation of columnar structures of the polymer oriented perpendicularly to the electrode surface. Recent studies from our group have provided correlations between the topography and electrochemical properties of thin supramolecular polymer layers [10]. The voltammetric and topographic features of spontaneously adsorbed $\left[\mathrm{Os}(\mathrm{bpy})_{2}(\mathrm{PVP})_{5} \mathrm{Cl}\right] \mathrm{Cl}$ (bpy = 2,2' -bipyridyl and $\mathrm{PVP}=\operatorname{poly}(4$-vinylpyridine $)$ ) on highly oriented pyrolitic graphite (HOPG) are illustrated in Fig. 2. Films exhibiting sharp voltammetric features (FWHM $<90 \mathrm{mV}$ ) are associated with molecular scale corrugation, suggesting that the PVP units tend to maximize their interactions at the adsorbed layer. Films featuring rougher topography yield broader voltammetric waves and larger peak-to-peak separation characteristic of slow charge transport phenomena. On the other hand, adsorption of the complex from diluted ethanolic solution leads to the formation of discrete nanosized aggregates at the HOPG surface as illustrated in Fig. 3. Our preliminary observations reveal that the topography of these aggregates is independent of the redox state of the polymer as probed by in situ acoustic SFM. This result suggests that the flux of ions associated with the transition from Os(II) to Os(III) does not involve significant swelling of the supramolecular aggregates at this dimension.

Another illustrative example of the morphological information that can be extracted from SFM images is given by the recent studies on ion intercalation into the graphene layers of HOPG electrodes [11-13]. Fig. 4 illustrates the difference in height of a HOPG step before and after $\mathrm{Li}^{+}$ intercalation at negative potentials. After a potentiodynamic cycle into the intercalation region, the step height increases and a thin film exhibiting blister-like features is generated at the HOPG surface. This film corresponds to the so-called solid electrolyte interphase, which arises from decomposition of the solvent in the intercalation potential range. Campana et al. employed a statistical analysis in order to minimize the effect of surface roughness on the estimation of the step height [11]. The average swelling of the HOPG upon intercalation was estimated to be $17 \pm 5 \%$.

\section{High-resolution Imaging of Electrode Reactivity}

The previous examples demonstrate the tremendous capabilities of SFM for mapping the morphological aspects of modified electrodes. However, this approach offers very little information in relation to local surface reactivity. A well-established technique for mapping electrochemical reactivity is scanning electrochemical microscopy (SECM), in which an ultramicroelectrode (UME) of dimensions in the range $0.1-10 \mu \mathrm{m}$ is used as a probe [14-16]. As schematically illustrated in Fig. 5A, the UME monitors the local concentration of a redox active species in the vicinity of the electrode surface. The amperometric or potentiometric signal can be measured as function of the distance $(d)$ from the electrode, the so-called approach curves, or as scanned across the surface at a constant height. In a loose analogy to STM, the distance between the UME and the surface is controlled by an electrochemical feedback system. The potential of the substrate and the UME are independently controlled by a bi-potentiostat. The imaging 

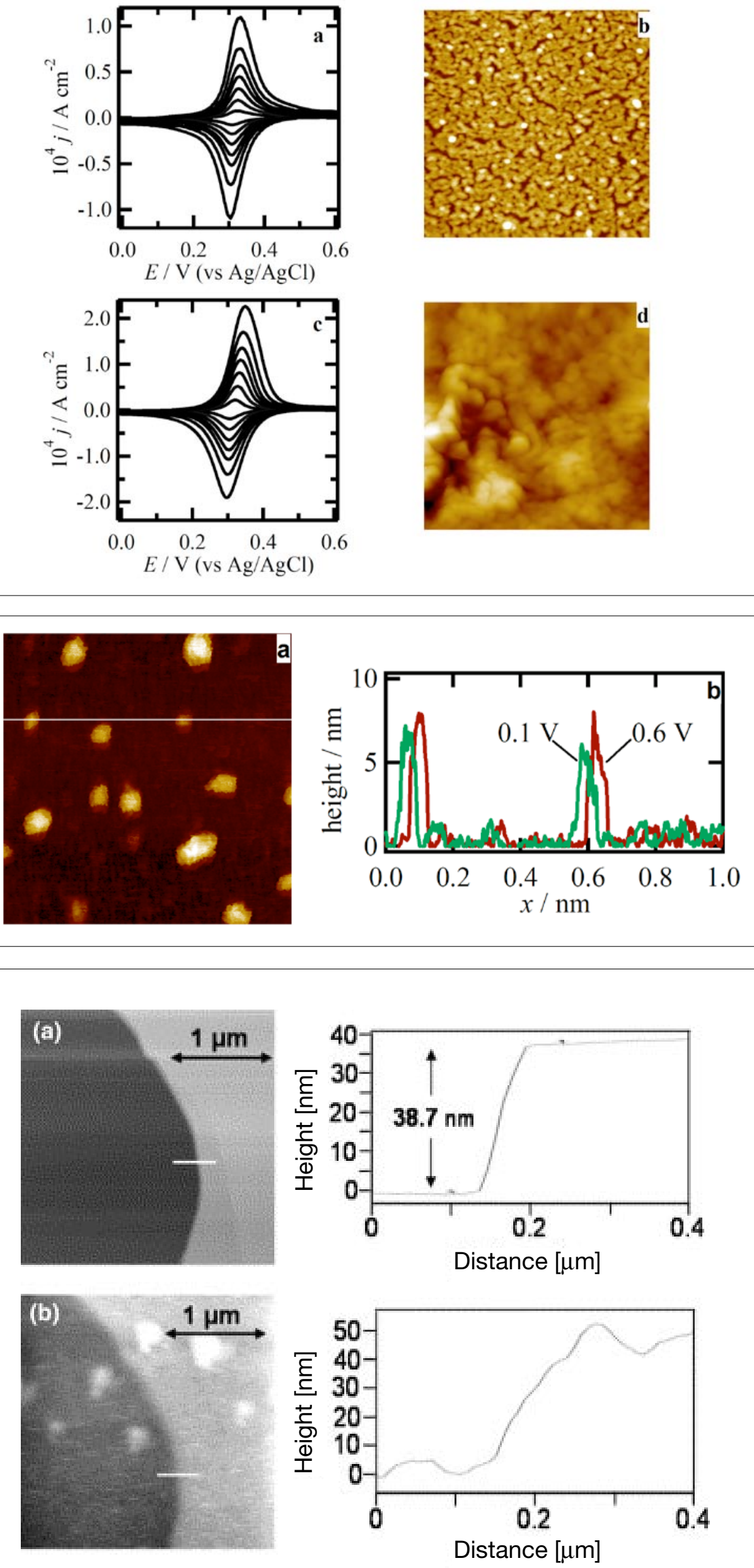

Fig. 2. Voltammetric and topographic features of spontaneously adsorbed $\left[\mathrm{Os}(\mathrm{bpy})_{2}(\mathrm{PVP})_{5} \mathrm{Cl}\right] \mathrm{Cl}$ complex on HOPG electrodes upon exposure to diluted aqueous solution for $(a, b) 1800 \mathrm{~s}$ and $(\mathrm{c}, \mathrm{d}) 4500 \mathrm{~s}$. The voltammograms in (a) were recorded at 10, 20,30, 40, 50, 70 and $100 \mathrm{mV} \mathrm{s}^{-1}$, showing well-defined narrow bands characteristic of a homogeneous phase formation involving attractive forces between adsorbates. The $1 \mu \mathrm{m} \times 1 \mu \mathrm{m} \times 2.5 \mathrm{~nm}$ image in (b) exhibits a molecularly flat polymer phase which homogeneously covers the electrode surface. The prominent globular features are of the same dimensions of the Os complex itself. Longer adsorption times induce a broadening of the voltammetric features (c) and an increase in the peak-to-peak separation. The changes in the voltammograms are linked to the generation of a more heterogeneous and disordered layer as confirmed by the $1 \mu \mathrm{m} \times 1 \mu \mathrm{m} \times 60 \mathrm{~nm}$ AFM image in (d). Reprinted from ref. [10]. Copyright (2006) with permission from Elsevier.

Fig. 3. Nanometre-sized aggregates of the $\left[\mathrm{Os}(\mathrm{bpy})_{2}(\mathrm{PVP})_{5} \mathrm{Cl}\right] \mathrm{Cl}$ complex generated by spontaneous adsorption from ethanolic solutions. The $1 \mu \mathrm{m} \times 1 \mu \mathrm{m}$ AFM image (a) shows the presence of a discrete polymer aggregates scattered across the HOPG surface. The height profile obtained for aggregates in the reduced and oxidized states (b) suggests that no significant morphological changes are observed upon modifying the redox state of the nanosized polymer phase.
Fig. 4. In situ contact mode SFM images of HOPG in ethylene carbonate/dimethyl carbonate solution containing $\mathrm{LiClO}_{4}$ at $3 \mathrm{~V}$ vs $E_{L \mathrm{O} / \mathrm{L}+}^{+}$before (a) and after (b) $\mathrm{Li}^{+}$intercalation. The image sizes are $6.25 \mu \mathrm{m}^{2}$ with a z-range of $120 \mathrm{~nm}$ for (a) and $140 \mathrm{~nm}$ (b). After a potentiodynamic cycle into the intercalation range, the step height and surface corrugation increased. The blister-like features are associated with the so-called solid electrolyte interphase. Reprinted from ref. [11]. Copyright (2005) with permission from Elsevier. 
resolution is conventionally determined by the electrode dimensions, i.e. several orders of magnitude lower than typical SFM topographic resolution.

Macpherson and Unwin developed a reliable approach for combining SECM and SFM to obtain sub-micron resolution of the reactivity of electrodes [17]. This remarkable achievement was based on the fabrication of sharp metallic tips carefully insulated except at the very end as exemplified in Fig. 5B. The tip is subsequently attached to a cantilever and mounted in the SFM scanner. More recently, other methods have been developed based on micro-fabrication techniques [18-21] and single-walled carbon nanotube (SWNT) templated metal probes [22]. Micrographs of some of these tips are illustrated in Figs 5C-D.

The outstanding spatial resolution achieved with SFM-SECM is exemplified in Fig. 6 where a $2-\mu \mathrm{m}$ Pt electrode is imaged employing a SWNT templated nanowire as probe [22]. High topographic resolution was obtained with these probes in tapping as well as in contact mode SFM (Fig. 6a). The current image was measured by setting the potential of the substrate electrode to reduce $\mathrm{Ru}\left(\mathrm{NH}_{3}\right)_{6}{ }^{3+}$ to $\mathrm{Ru}\left(\mathrm{NH}_{3}\right)_{6}{ }^{2+}$ at a diffusion controlled rate, while the tip was set to a potential for the oxidation of $\mathrm{Ru}\left(\mathrm{NH}_{3}\right)_{6}{ }^{2+}$. As seen in Fig. 6b, the current reaches a maximum on top of the $\mathrm{Pt}$ electrode, decreasing to zero as the probe is scanned over the insulating substrate. The similarity between the topographic and current images clearly indicates that the electrochemical response is determined by the dimensions of the Pt substrate electrode rather than the probe size. The representation in Fig. $6 \mathrm{c}$ illustrates the emergence of radial diffusional bands from the Pt micro-

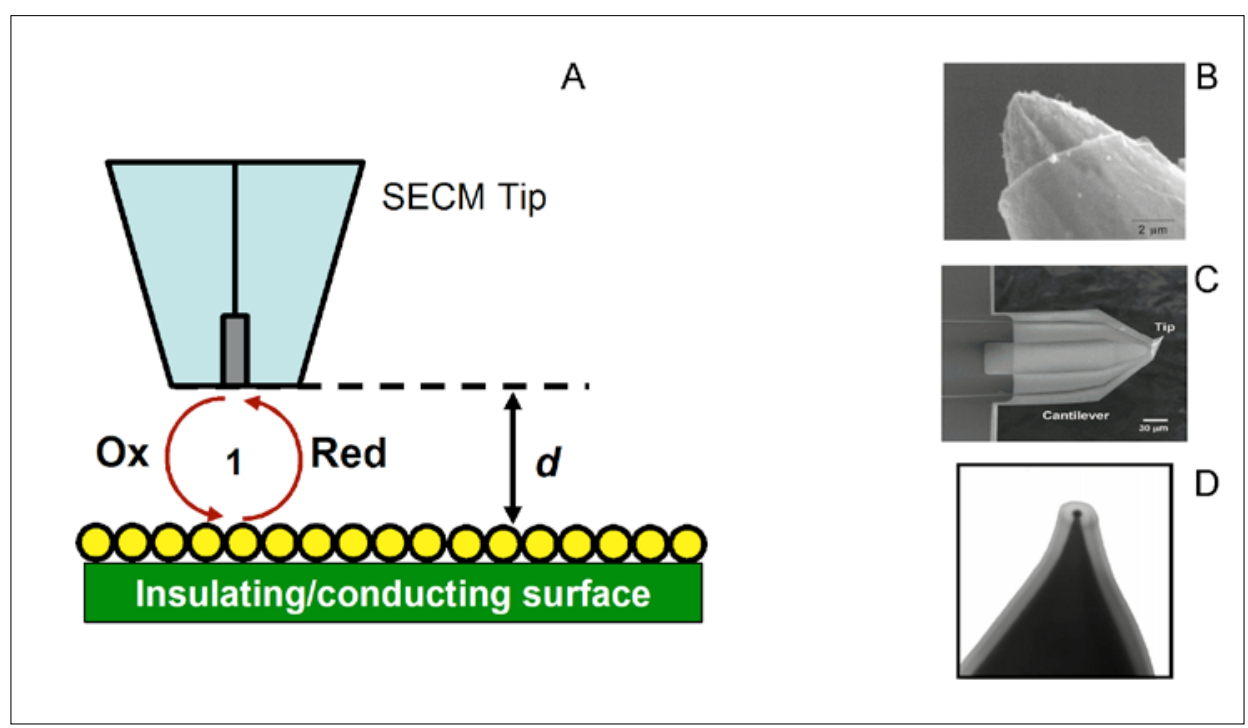

Fig. 5. Schematic representation of the electrochemical feedback configuration characteristic of SECM (A). Perturbation of the diffusion layer as the SECM tip approaches the surface will affect the current detected by the UME. For insulating substrates, the diffusion is hindered by the surface as the UME approaches generating a drop of the faradaic current (negative feedback). Fast regeneration of the redox species in conducting surfaces manifests itself by an increase of the faradaic current as the UME approaches (positive feedback). Metal coated SFM tips, thoroughly insulated except at the very end, allow operating conventional SFM instruments in SECM mode (B). Microfabricated tips generated by plasma enhanced chemical vapour deposition $(C)$ as well as single-walled carbon nanotube templated nanowires (D) have been successfully implemented as high resolution SECM tips. The image in (D) was obtained by focused ion beam (3.7 $\mu \mathrm{m}$ image width). Figs (B), (C) and (D) were reprinted with permission from ref. [17], [18] and [22], respectively. Copyright (2000 and 2005) American Chemical Society.

electrodes as expected theoretically, even if the shape of the electrode is not circular.

\section{Double Layer Forces at the Electrode/Electrolyte Interface}

As one of the key aspects of electrochemical process, the double layer has been subject of extensive experimental and the- oretical research. Classical electrochemical techniques and powerful molecular dynamic simulations have also provided detailed description of the double layer structure at well-defined interfaces [23][24]. Spectroscopic and diffraction methods including electrolyte electroreflectance, non-linear optics and in situ X-ray diffraction have also provided detailed aspects of the electrode/electrolyte interface, in particular the
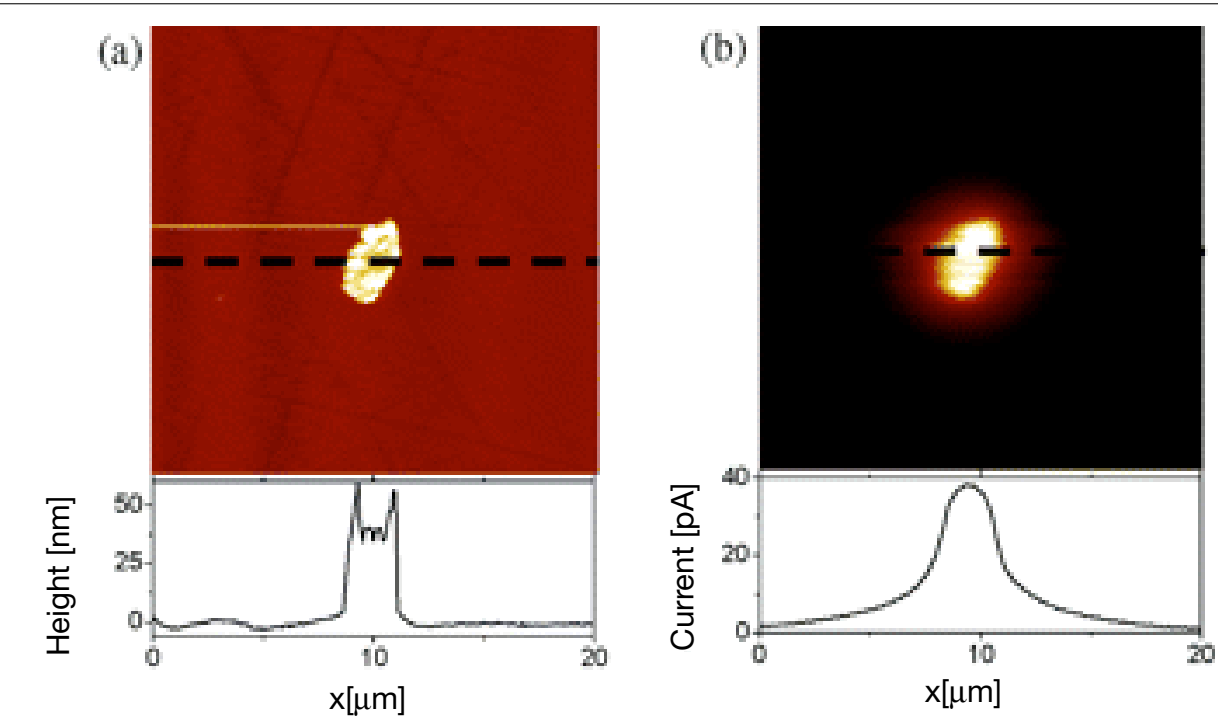

(C)
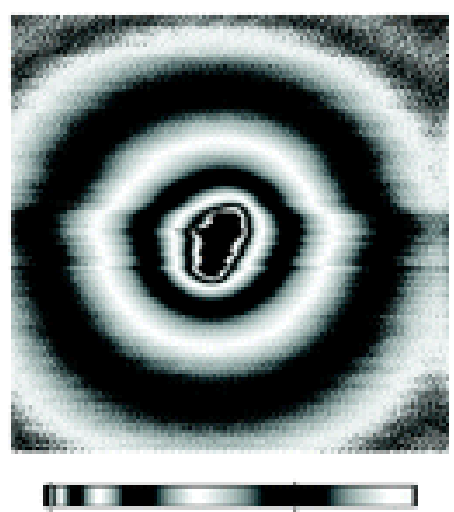

Fig. 6. Topographic SFM (a) and electrochemical (b,c) images of a $2-\mu \mathrm{m}$ Pt electrode recorded by SWNT templated nanowire in SECM-SFM configuration. Image (c) shows the radial diffusion profile of the redox species propagating from the electrode surface. The probe was scanned at $0.5 \mathrm{~Hz}$ and the image area was $20 \mu \mathrm{m}^{2}$. Reprinted with permission from ref. [22]. Copyright (2005) American Chemical Society. 
electronic structure of the electrode surface and specifically adsorbed species [25][26]. The introduction of SFM-based techniques to measure the forces associated with the double layer has provided valuable new perspectives to tackle this old concept, which is central not only to electrochemistry, but also to colloidal science and biological membrane processes.

The typical kind of tips used in these measurements consists of triangular shaped cantilevers modified with a spherical silica bead. The diameter of the silica bead, which is carefully glued to the apex of the cantilever, is commonly between 10 and $60 \mu \mathrm{m}$. Although the relatively large features of the tip hinder the spatial resolution of the force measurements, the well-defined geometry allows accurate determination of the magnitude of the double layer forces at planar surfaces. The illustrative review by Butt highlights some of the key issues in connection to tip preparation [27].

The interaction between two charged surfaces can be described by the theory of Derjaguin-Landau-Verwey-Overbeek (DLVO). In this theory, the effective force is essentially determined by attractive van der Waals (VA) and repulsive/attractive electrostatic interactions (VE). For the case of spheres of radius RT, the force can be simply expressed as in Eqn. (1) [28].

$$
F=2 \pi R_{\mathrm{T}}\left(V_{\mathrm{A}}+V_{\mathrm{E}}\right)
$$

The van der Waals term can be described in terms of the Hamaker constant $A_{\mathrm{H}}$

$$
V_{\mathrm{A}}=-A_{\mathrm{H}} / 12 \pi d^{2}
$$

where $d$ is the separating distance between the spheres. The constant $A_{\mathrm{H}}$ can be determined experimentally by distance-force measurements in systems mostly determined by van der Waals attractions, e.g. in air and highly purified water. On the other hand, the electrostatic terms can be evaluated from integration of the electrostatic force. In the case of 1:1 electrolytes, the electrostatic interaction is given by [28],

$$
\begin{aligned}
V_{\mathrm{E}}= & -\int_{\infty}^{d}\left\{2 n^{\circ} k T\left[\cosh \left(\frac{z e \psi}{k T}\right)-1\right]\right. \\
& \left.-\frac{\varepsilon}{2}\left(\frac{\mathrm{d} \psi}{\mathrm{d} z}\right)\right\} \mathrm{d} z
\end{aligned}
$$

where $n^{\circ}$ and $\varepsilon$ correspond to the concentration of the electrolyte and the dielectric constant of the medium. The electrostatic potential $\psi$ is given by the solution of the Poisson-Boltzmann Eqn

$$
\frac{\mathrm{d}^{2} \psi}{\mathrm{dz}^{2}}=-\frac{1}{\varepsilon} \sum_{\mathrm{i}} e n_{\mathrm{i}}^{\circ} z_{\mathrm{i}} \exp \left(-\frac{z_{\mathrm{i}} e \psi}{k T}\right)
$$

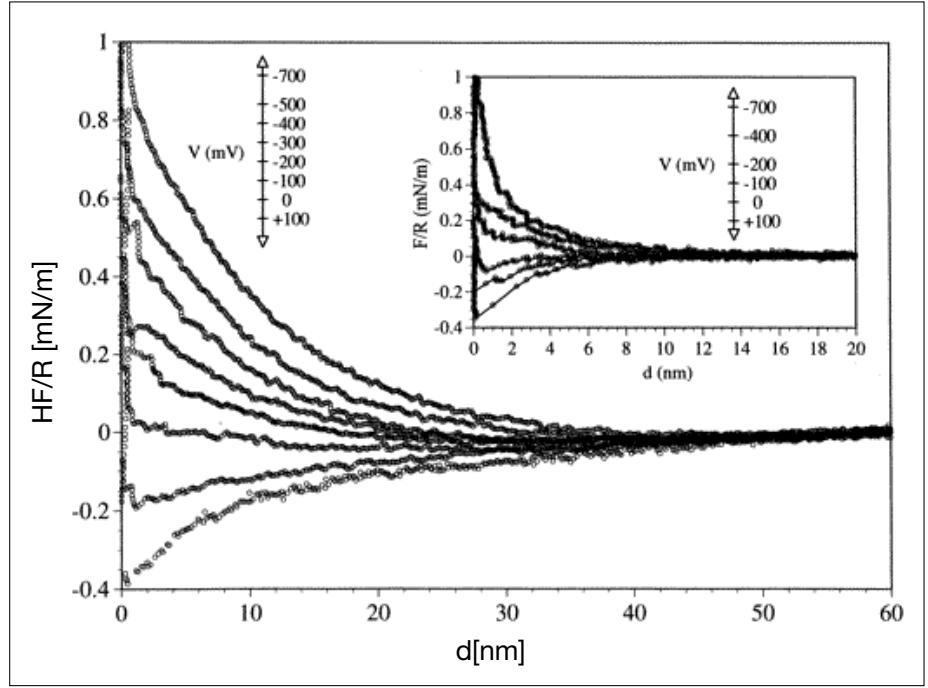

Fig. 7. Force-distance curves between a silica sphere and a gold electrode in an aqueous solution of $10^{-3} \mathrm{~mol} \mathrm{dm}^{-3} \mathrm{KCl}$ at $298 \mathrm{~K}$ and pH 5.5 as a function of the applied potential at the Au electrode. The curves evolves from a repulsive behaviour between both surfaces at negative potentials (positive forces), to a attractive regime at positive potentials. The potential scale is referred to the standard calomel electrode (SCE). The inset shows the same family of curves but for an electrolyte concentration of $10^{-2} \mathrm{~mol}$ $\mathrm{dm}^{-3}$. Reprinted with permission from ref. [28]. Copyright (1996) American Chemical Society.

Hillier et al. numerically solved the complete non-linear form of Eqn. (4) in order to accurately describe force-distance curves as a function of the electrode potential [28]. Fig. 7 illustrates the effect of the applied potential on the forces between a gold electrode and a silica sphere in a $\mathrm{KCl}$-containing electrolyte at $\mathrm{pH}$ 5.5. As the silica sphere is negatively charged at this $\mathrm{pH}$, the force-distance curve is dominated by repulsive forces at negative potentials. At positive potentials, the responses are dominated by the attractive electrostatic interactions. The onset of the distance dependence force in $\mathrm{KCl}$ of concentration $10^{-3} \mathrm{~mol} \mathrm{dm}{ }^{-3}$ is located around $40 \mathrm{~nm}$, decreasing to less than $10 \mathrm{~nm}$ for concentrations of $10^{-2} \mathrm{~mol}$ $\mathrm{dm}^{-3}$. The transition from attractive to repulsive regime, the potential of zero force $(p z f)$, was found dependent on the nature of the anion in solution. The behaviour of the $p z f$ was consistent with the potential of zero charge ( $p z c)$ obtained from double layer capacitance measurements.

Studies of the double layer forces revealed fundamental information that cannot be obtained from classical electrochemical data. For instance, Wang and Bard demonstrated that the classical Goy-ChapmanStern model (GCS), widely used for describing the charge of the diffuse electrical layer, is inadequate even at the metal/electrolyte interface in the absence of specific adsorption [29]. The experimental determination of the diffuse layer force allowed estimation of the effective diffuse layer charge which was only $10 \%$ of the real charge obtained from the capacitance data. The surprisingly small effective charge is a result of ion correlation/ion condensation effects which are not included in the GCS. Other interesting observations by this method involve the structure of the double layer at electrode surfaces modified by conducting polymers [30]. The independence of the force-distance curve on the applied potentials for polypyrrole and polythiophene modified electrodes demonstrated the absence of a diffuse layer in these systems. These results provide very valuable information in the interpretation of electrochemical responses at complex interfacial systems.

\section{Concluding Remarks}

The growing field of electrochemistry at modified electrodes tremendously relies on techniques capable of providing detailed pictures of the structure and properties of the surface at the nanoscale. While atomic scale resolution remains almost exclusively accessible by STM, techniques based on SFM exhibit a superior versatility and adaptability to different types of sample. In this overview, it is shown that SFM can provide not only nanoscale mapping of topography, but also of electrochemical reactivity and double layer forces at electrochemical interfaces. The information provided by these new approaches has certainly enhanced our current understanding of structure-reactivity relationships at highly complex interfaces.

Most of these approaches have already been implemented in commercially avail- 
able instruments. Furthermore, the possibility of micro-fabricating tips for SECMSFM and double layer force measurements will make these approaches more widely accessible. Chemical modification of tips in order to enhance the contrast with different functionalities at the electrode surface is certainly an upcoming development in this field. A high profile example of this approach is given by the direct quantification of the chemical forces involving host-guest interactions [31][32]. Another interesting on-going development is the determination of molecular nanowire conductance as a function of the applied force. For instance, the work by Porat and co-workers has led to a series of fascinating discoveries in the highly controversial field of DNA conductivity [33].

\section{Acknowledgements}

DJF is grateful to Prof. Hans Siegenthaler from the Universität Bern for discussions and an advanced copy of ref. 13. Prof. J.G. Vos from Dublin City University is also acknowlegded for kindly providing the $\left[\mathrm{Os}(\mathrm{bpy})_{2}\left(\mathrm{PVP}_{5}\right] \mathrm{Cl}\right.$ complex. Evren Aslan and Niklaus Baumann are gratefully acknowledged for providing the experimental results shown in Fig. 3. The financial support by the Swiss National Science Foundation (projects PP002-68708 and 200021105238 ) is also acknowledged.

Received: September 21, 2006

[1] A.A. Gewirth, B.K. Niece, in 'Electrochemical Nanotechnology: In situ Local Probing at Electrochemical Interfaces', Eds W.J. Lorenz, W. Plieth, Wiley-VCH, Weinheim, 1998, p. 113.

[2] E. Weilandt, A. Menck, M. Binggeli, O. Marti, in 'Nanoscale Probes of the Solid/ Liquid Interfaces', Eds A.A. Gewirth, H. Siegenthaler, Kluwer Academic Publisher, Dordrecht, 1995, p. 307.

[3] W.A. Hayes, H. Kim, X.H. Yue, S.S. Perry, C. Shannon, Langmuir 1997, 13, 2511.

[4] H.U. Krotil, T. Stifter, H. Waschipky, K. Weishaupt, S. Hild, O. Marti, Surf. Interface Anal. 1999, 27, 336.
[5] P.D. Ashby, C.M. Lieber, J. Am. Chem Soc. 2005, 127, 6814.

[6] S. Manne, P.K. Hansma, J. Massie, V.B. Elings, A.A. Gewirth, Science 1991, 251 183.

[7] C.A. Alves, M.D. Porter, Langmuir 1993, 9, 3507.

[8] P.I. Oden, N.J. Tao, S.M. Lindsay, J. Vac Sci. Technol. B 1993, 11, 137.

[9] K. Naoi, Y. Oura, Y. Iwamizu, N. Oyama, J. Electrochem. Soc. 1995, 142, 354.

[10] E. Aslan, F.P. Campana, D.J. Fermín, H. Siegenthaler, F. Frehill, J.G. Vos, J. Electroanal. Chem., in press.

[11] F.P. Campana, R. Kotz, J. Vetter, P. Novak, H. Siegenthaler, Electrochem. Commun. 2005, 7, 107.

[12] G.G. Wildgoose, M.E. Hyde, N.S. Lawrence, H.C. Leventis, L. Jiang, T.G.J. Jones, R.G. Compton, Langmuir 2005, 21, 4584.

[13] F.P. Campana, M. Hahn, A. Foelske, P. Ruch, R. Kötz, H. Siegenthaler, Electrochem. Comm. 2006, 8, 1363.

[14] J.V. Macpherson, P.R. Unwin, J. Phys. Chem. 1996, 100, 19475.

[15] J.V. Macpherson, J.P.G. de Mussy, J.L. Delplancke, J. Electrochem. Soc. 2002 , 149, B306.

[16] A.J. Bard, F.R.F. Fan, D.T. Pierce, P.R. Unwin, D.O. Wipf, F.M. Zhou, Science 1991, 254, 68.

[17] J.V. Macpherson, P.R. Unwin, Anal. Chem. 2000, 72, 276

[18] P.S. Dobson, J.M.R. Weaver, M.N. Holder, P.R. Unwin, J.V. Macpherson, Anal. Chem. 2005, 77, 424.

[19] A. Kueng, C. Kranz, A. Lugstein, E. Bertagnolli, B. Mizaikoff, Angew. Chem. Int. Ed. 2003, 42, 3238.

[20] P.L.T.M. Frederix, M.R. Gullo, T. Akiyama, A. Tonin, N.F. de Rooij, U. Staufer, A. Engel, Nanotechnology 2005, 16, 997.

[21] P.S. Dobson, J.M.R. Weaver, D.P. Burt, M.N. Holder, N.R. Wilson, P.R. Unwin, J.V. Macpherson, Phys. Chem. Chem. Phys. 2006, 8, 3909.

[22] D.P. Burt, N.R. Wilson, J.M.R. Weaver, P.S. Dobson, J.V. Macpherson, Nano Lett 2005, 5, 639 .
[23] A.A. Kornyshev, E. Spohr, M.A. Vorotyntsev, in 'Encyclopedia of Electrochemistry. Volume 1. Thermodynamics and Electrified Interfaces', Eds E. Gileadi, M. Urbakh, Wiley-VCH, Weinheim, 2002, p 33.

[24] W. Schmickler, in 'Encyclopedia of Electrochemistry. Volume 1. Thermodynamics and Electrified Interfaces', Eds E. Gileadi, M. Urbakh, Wiley-VCH, Weinheim, 2002 p. 133.

[25] W. Schmickler, Chem. Rev. 1996, 96, 3177.

[26] See articles in 'Advances in Electrochemical Science and Engineering. Volume 9. Diffraction and Spectroscopic Methods in Electrochemistry', Eds R.C. Alkire, D.M. Kolb, J. Lipkowski, P.N. Ross, WileyVCH, Weinheim, 2006.

[27] H.J. Butt, in 'Encyclopedia of Electrochemistry. Volume 1. Thermodynamics and Electrified Interfaces', Eds E. Gileadi, M. Urbakh, Wiley-VCH, Weinheim, 2002, p. 225.

[28] A.C. Hillier, S. Kim, A.J. Bard, J. Phys. Chem. 1996, 100, 18808.

[29] J. Wang, A.J. Bard, J. Phys. Chem. B 2001, 105, 5217

[30] J. Wang, A.J. Bard, J. Am. Chem. Soc. 2001, 123, 498.

[31] T. Auletta, M.R. de Jong, A. Mulder, F. van Veggel, J. Huskens, D.N. Reinhoudt, S. Zou, S. Zapotoczny, H. Schonherr, G.J. Vancso, L. Kuipers, J. Am. Chem. Soc. 2004, 126, 1577 .

[32] S. Zapotoczny, T. Auletta, M.R. de Jong, H. Schonherr, J. Huskens, F. van Veggel, D.N. Reinhoudt, G.J. Vancso, Langmuir 2002, 18, 6988 .

[33] H. Cohen, C. Nogues, D. Ullien, S. Daube, R. Naaman, D. Porath, Faraday Discuss. 2006, $131,367$. 